\title{
Object memory and change detection: Dissociation as a function of visual and conceptual similarity
}

\author{
Yei-Yu Yeh *, Cheng-Ta Yang \\ Department of Psychology, National Taiwan University, No. 1, Section 4, Roosevelt Road, Taipei 106, Taiwan
}

Received 30 July 2006; received in revised form 3 March 2007; accepted 8 March 2007

Available online 26 April 2007

\begin{abstract}
People often fail to detect a change between two visual scenes, a phenomenon referred to as change blindness. This study investigates how a post-change object's similarity to the pre-change object influences memory of the pre-change object and affects change detection. The results of Experiment 1 showed that similarity lowered detection sensitivity but did not affect the speed of identifying the pre-change object, suggesting that similarity between the pre- and post-change objects does not degrade the pre-change representation. Identification speed for the pre-change object was faster than naming the new object regardless of detection accuracy. Similarity also decreased detection sensitivity in Experiment 2 but improved the recognition of the pre-change object under both correct detection and detection failure. The similarity effect on recognition was greatly reduced when $20 \%$ of each pre-change stimulus was masked by random dots in Experiment 3. Together the results suggest that the level of pre-change representation under detection failure is equivalent to the level under correct detection and that the pre-change representation is almost complete. Similarity lowers detection sensitivity but improves explicit access in recognition. Dissociation arises between recognition and change detection as the two judgments rely on the match-to-mismatch signal and mismatch-to-match signal, respectively.
\end{abstract}

(C) 2007 Elsevier B.V. All rights reserved.

PsycINFO classification: 2323

Keywords: Change blindness; Object memory; Similarity effect; Visual perception

\section{Introduction}

The visual environment changes over time. Old buildings are torn down and new ones are constructed at the same location. As new things replace old, what happens to the memory of the old? How do people remember what was previously at a particular location while viewing something new? Answers to these two questions reflect how people represent and remember visual information in an ever-changing world.

The nature of visual representation has been investigated in various contexts. Results from studies using the method of rapid stimulus presentation suggest that detailed

\footnotetext{
* Corresponding author. Tel.: +88622368 8837.

E-mail address: yyy@ntu.edu.tw (Y.-Y. Yeh).
}

visual representation must be accumulated across saccades in eye movements (see Coltheart, 1999 for a review). Yet, the global semantic information can be quickly extracted as the theme of a natural scene can be rapidly categorized (Fabre-Thorpe, Delorme, Marlot, \& Thorpe, 2001; Mace, Thorpe, \& Fabre-Thorpe, 2005) and an object inconsistent to the scene gist can be recognized after a presentation of 150 ms (Hollingworth \& Henderson, 1999). Recent work on change blindness also highlights that observers may not form a complete representation when perceiving the visual world (e.g., Rensink, 2000 for a review; Simons \& Rensink, 2005 for discussion). Observers often failed to detect a change in natural scenes even after viewing the initial scene for $11.25 \mathrm{~s}$ before a mask replaced the image (Simons, Franconeri, \& Reimer, 2000).

Despite the failure in change detection, the details of a natural scene may be stored in visual representation as 
revealed by both indirect (Hollingworth, Williams, \& Henderson, 2001; Ryan \& Cohen, 2004; Silverman \& Mack, 2006) and direct measures (e.g., Beck \& Levin, 2003; Mitroff, Simons, \& Levin, 2004; Simons, Chabris, Schnur, \& Levin, 2002). The dissociation between change detection and memory performance based on intentional retrieval (Simons et al., 2002; Varakin \& Levin, 2006) is intriguing for three reasons. First, a change detection task requires multiple cognitive processes including encoding, memory maintenance and rehearsal, retrieval, comparison, and the decision process. Memory judgments also evoke these processes. Second, signal detection theory has been adopted to account for both change detection (Wilken \& Ma, 2004) and recognition of previously studied stimuli (e.g., Dunn, 2004; Gardiner, Konstantinou, Karayianni, \& Gregg, 2005; Hirshman, 1998; Smith \& Duncan, 2004). Similar processing algorithms may be adopted in both detection and memory performances. Third, change detection may influence memory representation as correct change detection leads to inhibition or attenuation of unchanged and pre-change information (Silverman \& Mack, 2006).

Given that people are often required to compare current percepts with memory, understanding the relationship between change blindness and memory representation can reveal how memory is accessed to build the on-line representation that corresponds to the visual perception (Hollingworth, 2004). The purpose of this study is to investigate the relationship between object memory and change detection. We focus on a specific issue: the impact of the post-change object on the memory of a pre-change object and on change detection. We demonstrate how object memory and change detection are dissociable.

\subsection{Aim of the present study}

To examine the influence of the post-change object on the memory of the pre-change object and on change detection, we manipulated the similarity between the pre- and post-change objects. If the presence of a post-change object overwrites the pre-change representation as the overwriting hypothesis (Beck \& Levin, 2003; see Simons, 2000) suggests, a similar post-change object should cause damage to the pre-change representation as the magnitude of backward masking is accentuated by the similarity between a target and the subsequent mask (see Enns \& Di Lollo, 2000). A similar post-change object should also decrease change detection performance as previous studies have shown (Mitroff, Simons, \& Franconeri, 2002; Silverman \& Mack, 2006; Smilek, Eastwood, \& Merikle, 2000; Williams \& Simons, 2000; Zelinsky, 2003). Change detection relies on the mismatch-to-match signal in the decision process as characterized by the signal detection framework (Wilken \& Ma, 2004).

An indirect test of memory was adopted in Experiment 1 using an object identification task to investigate the similarity effect on change detection and object memory. A direct test of memory was adopted in both Experiments 2 and 3 using a two-alternative forced-choice (2AFC) recognition task to examine the similarity effect. Unmasked pre-change stimuli were used in Experiment 2 and $20 \%$ of the prechange stimuli were masked by random dots in Experiment 3. If a similar post-change object causes more damage than a dissimilar one to the pre-change representation, we should observe a similarity cost on the memory of the pre-change object and masking should modulate the similarity effect. Object memory and change detection should not dissociate as a function of similarity between the preand post-change objects.

In contrast, the presence of a post-change object may not overwrite the pre-change representation. One source of change blindness is that nothing is compared between the pre- and post-change objects (Mitroff et al., 2004) or retrieval failure (Hollingworth, 2003). If nothing is compared between the pre- and post-change objects or the pre-change representation is not retrieved, whether the pre- and post-change objects are similar or not should not matter under detection failure. We should not observe a similarity effect on object identification in Experiment 1, especially under detection failure.

How can access to pre-change representation be improved if the pre-change object is stored in memory but not retrieved (Hollingworth, 2003) or is disconnected from consciousness (Ryan \& Cohen, 2004) under change blindness? On the basis of the signal detection framework, we predict that similarity of pre- and post-change objects should improve access to the pre-change representation and enhance memory recognition under both change blindness and correct detection in Experiment 2.

The signal used in memory recognition is the magnitude of the match between a test probe and memory traces. Similarity between the pre- and post-change objects should increase the magnitude of the match between a test probe and a pre-change object for two reasons. First, a similar post-change object provides retrieval cues for accessing the pre-change object. More of the information from the pre-change object can be used in matching the test probe and the pre-change object. Second, a similar post-change object also increases the match signal when the test probe is compared to all memory traces retained from the preand post-change stimulus arrays. According to global familiarity memory models (e.g., Hintzman, 1988; Murdock, 1997; Shiffrin \& Steyvers, 1997), a test probe is compared in parallel to all memory traces during recognition. A decision based on all the matches and mismatches from the comparisons influences familiarity and hence determines recognition performance. With similar pre- and post-change objects, the summed match signal from all comparisons should be stronger, producing greater familiarity in contrast to the condition in which the two objects are dissimilar. Through either a retrieval or matching mechanism, a similar post-change object should increase the match-to-mismatch signal to improve recognition of the pre-change object. As similarity lowers the mismatchto-match signal in change detection, similarity should lead 
to dissociation between change detection and recognition of the pre-change object in Experiment 2.

The result of importance is the similarity effect on object memory and change detection when pre-change stimuli are masked by random dots in Experiment 3. Masking should decrease the visual information that can be used in change detection. Detection performance should decrease as compared to the performance observed in Experiment 2. Masking should also lower the match-to-mismatch signal in the memory decision and hence reduce the similarity benefit. The critical evidence is the degree to which the similarity benefit is reduced. If no similarity benefit is observed in Experiment 3, the contrast with the results of Experiment 2 would suggest that the similarity benefit under change blindness requires an almost complete pre-change representation.

Although previous studies have demonstrated that visual representation may exist under change blindness, recognition (Hollingworth, 2004; Mitroff et al., 2004) and cued recall (Simons et al., 2002) are results of intentional retrieval that might be subject to memory editing (Odegard \& Lampinen, 2006). Findings based on eye movements (Hollingworth et al., 2001; Ryan \& Cohen, 2004) might be subject to the argument that eye movement to a location does not necessarily indicate the existence of visual representation. Attention to a location can be captured by stimulus salience (Scholl, 2000), and feature salience correlates with eye movements in free viewing of natural scenes (Parkhurst, Law, \& Neibur, 2002). Moreover, these results do not necessarily indicate that the pre-change representation is almost complete as a partial pre-change representation can lead to the same results.

The impact of change magnitude on change detection has been thoroughly investigated (Mitroff et al., 2002; Silverman \& Mack, 2006; Smilek et al., 2000; Williams \& Simons, 2000; Zelinsky, 2003). In contrast, the effect of similarity on object memory in the context of change detection has never been addressed. By examining the similarity effect on object memory and change detection, the results of these three experiments should provide evidence for two important issues: the completeness of the pre-change representation under change blindness and under correct detection, and the reason that object memory based on intentional retrieval and change detection are dissociable.

\section{Experiment 1}

The objective of this experiment was to investigate how a similar post-change object can influence the pre-change representation via an indirect test of memory. A priori hypotheses predict different patterns of results. The overwriting hypothesis (Beck \& Levin, 2003; see Simons, 2000) predicts a detriment to the pre-change representation due to similarity; similarity lowers detection performance. The hypothesis that nothing is compared (Mitroff et al., 2004) or retrieval failure (Hollingworth, 2003) predicts that similarity should not affect pre-change representation when the pre-change object is not compared or retrieved under change blindness.

A recent study used an indirect test of memory to investigate pre-change representations (Silverman \& Mack, 2006). In their Experiment 3, letters in any row of a $3 \times 3$ matrix could change in one, two, or all three positions. The results showed that all stimuli including the unchanged, pre-change, and post-change letters led to repetition priming under detection failure, but only postchange stimuli produced repetition priming under correct detection. The findings suggest that the visual system inhibits or attenuates all other stimulus information to protect the changed input and maximize change detection.

The observation that pre-change representations are attenuated after successful retrieval and comparison is intriguing as it suggests that correct detection can degrade memory representations. However, participants in Silverman and Mack's (2006) Experiments, 3a and 3b, respectively, showed an average of $60 \%$ and $61 \%$ correct identification of the pre-change and post-change stimuli under correct detection. According to their inhibition/ attenuation hypothesis, identification accuracy for the post-change stimuli should be higher than accuracy for the pre-change stimuli under correct detection if the prechange representations were inhibited or attenuated to protect the changed information. Given that different groups of participants served in these two experiments, it is unclear whether the predicted difference would be observed in a within-subjects design.

We extended Silverman and Mack's (2006) work by manipulating visual and conceptual similarity between the pre- and post-change objects as an object is characterized by both visual and semantic properties (Boucart \& Humphreys, 1994; Hollingworth \& Henderson, 2002), which are subserved by separate neural mechanisms (Badgaiyan, 2000; Pins, Meyer, Foucher, Humphreys, \& Boucart, 2004). Evidence also shows that the semantic theme of a scene can be categorized in the near absence of attention (Li, VanRullen, Koch, \& Perona, 2002).

We tested the memory of the pre-change object by modifying the continuous identification task used in a study of repetition priming of non-words, as the repetition priming effect reflects a form of implicit memory (Stark \& McClelland, 2000). We investigated the similarity effect on object identification. We also examined whether the representation of the pre-change object supports a priming effect under both detection failure and correct detection. If the visual system inhibits or attenuates visual representation of a pre-change object for correct detection (Silverman \& Mack, 2006), repetition priming should not be observed for the pre-change object under correct detection.

\subsection{Method}

\subsubsection{Participants}

Sixteen undergraduate students at National Taiwan University participated in this one-hour experiment, with 
14 receiving a bonus credit for an introductory psychology course and two receiving NT\$150 (about US\$5) for their participation. They were all aged $18-27$ with normal or corrected-to-normal vision.

\subsubsection{Equipment, stimuli, and design}

A PC with a $3.30 \mathrm{GHz}$ Intel Pentium IV processor was used to run the experiment. The display monitor used was a 17 -in. color monitor with a vertical refresh rate of $75 \mathrm{~Hz}$. E-Prime (Schneider, Eschman, \& Zuccolotto, 2002) was used to run the experiment.

Three hundred and twelve color images from the CorelDraw v5.0 (CorelDRAW!, 1994) art library were used as object stimuli. Each object was converted to a 24-level color scale and sized to a maximum of 120 pixels on both the $x$ and $y$ dimensions. Each object subtended a visual angle of $5.24^{\circ}$ (horizontal) $\times 4.29^{\circ}$ (vertical) at the viewing distance of approximately $60 \mathrm{~cm}$. Six stimuli were randomly placed around an imaginary circle with a diameter of $7.01^{\circ}$ on each trial. A white background was used throughout the trial.

Only one object in the array changed in the change trials, with a post-change target replacing the pre-change target. The relationship between the pre- and post-change targets was manipulated into four categories: (1) visually and conceptually dissimilar, (2) visually similar and conceptually dissimilar, (3) visually dissimilar and conceptually similar, and (4) visually and conceptually similar. Visual similarity was defined by similarity in color, size, shape, orientation, or a combination of these attributes. Conceptual similarity was defined as categorical similarity with categories selected from Battig and Montague's
(1969) category norms. We also combined subordinate categories in the norms. For example, four-legged animals and birds were combined into the superordinate category of animals. The results of a rating study (Yang, Hung, \& Yeh, 2003) confirmed a two-dimensional structure of object similarity and were used to select the stimuli for this study. Fig. 1 shows a pair of examples from each of the four clusters and the mean rating results.

Two hundred and fifty-two experimental trials were constructed with a 2:1 ratio between the change and no-change trials. After selecting the pre- and post-change targets, the unchanged objects in the change trials were randomly selected from 310 objects. The stimuli in the no-change trials were randomly selected from the whole set of 312 stimuli.

Of the 168 change trials, half consisted of target pairs that were visually and conceptually dissimilar. A 1:1 ratio between similar and dissimilar pre- and post-change pairs was used so that participants would not be biased toward relating the two targets by similarity. For the other three conditions, we randomly selected 28 stimuli from each category. An object identification task was performed after change detection on every trial. After a no-change trial, an unchanged object was randomly selected from the six stimuli for identification. After a change trial, either the pre-change target or a new object was presented for identification. The ease of identification between the pre-change target and the new object was matched. The ratio was $4: 3$ between the two types of test probes across all the change trials. This ratio was adopted to approximate equal frequency but allow more data points for testing the prechange target. The trial composition was randomly mixed in the experiment.

\begin{tabular}{|c|c|c|c|c|}
\hline \multicolumn{5}{|c|}{ Similarity } \\
\hline Visual & Low & Low & High & High \\
\hline Pre-change & Low & High & Low & High \\
\hline Post-change & & & & \\
\hline Visual similarity & 1.35 & 2.52 & 3.07 & 4.61 \\
Conceptual similarity & 1.25 & 5.04 & 1.60 & 5.26 \\
\hline
\end{tabular}

Fig. 1. An example of pre- and post-change targets in the four conditions derived from the manipulation of visual and conceptual similarity. The mean ratings based on a 7-point Likert type scale are also presented. 


\subsubsection{Procedure}

Participants were asked to be as accurate as possible. Speed was not emphasized for change detection. Participants were also told that an identification task would occur after the detection task. Six practice trials were run for participants to become familiar with the task. To suppress verbal rehearsal, at the beginning of each block participants

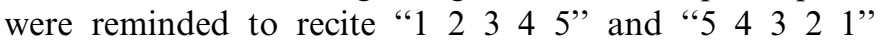
throughout the trial until the probe display was shown (see Fig. 2). A fixation cross was shown for $1000 \mathrm{~ms}$ at the beginning of each trial. After the fixation cross, the pre-change display was presented for $2000 \mathrm{~ms}$. A blank screen was displayed during the $350 \mathrm{~ms}$ retention interval. The post-change display was presented afterward for $2000 \mathrm{~ms}$. After another interval of $350 \mathrm{~ms}$, a mask display was presented. A Mondrian pattern was shown at each stimulus location to mask the post-change stimuli for $2000 \mathrm{~ms}$. Although a mask display has rarely been used in previous studies of change blindness with a one-shot paradigm, this method was adopted so that the encoding and retention intervals would be identical for both the pre- and post-change arrays with the presence of a subsequent stimulus display.

After the mask display, a probe display required participants to judge whether there was any change between the pre- and post-change arrays. The object identification task was then performed. The identification task followed the identification phase of Stark and McClelland's (2000) procedure (see Fig. 3) with one modification. We used a colored Mondrian pattern rather than a row of \# signs on each stimulus. A test object appeared initially for $10 \mathrm{~ms}$, and was followed by the mask display for a duration of
$240 \mathrm{~ms}$. If the participants could not name the object, the duration increased another $10 \mathrm{~ms}$ for the object and decreased $10 \mathrm{~ms}$ for the mask to keep the total viewing time constant at $250 \mathrm{~ms}$ for each test cycle. The test cycles were repeated until the participant named the object or for $4200 \mathrm{~ms}$.

Participants were told that a test object would appear on the screen for a very brief duration and then it would immediately be masked. The object would reappear and continue to flash on the screen for increasing durations, alternating with the mask, and become clearer with time. Participants were asked to name the object aloud as soon as they could identify it. They were told to do it as accurately and as quickly as possible. The voice onset terminated the identification task with reaction time recorded and the experimenter coded naming responses. No feedback was provided on the correctness of change detection or naming responses.

\subsection{Results}

\subsubsection{Change detection}

We conducted a 2 (visual similarity) $\times 2$ (conceptual similarity) repeated measures analysis of variance (ANOVA) on detection performance, with the effect size $\eta^{2}$ defined by the $\mathrm{SS}($ effect)/[SS(effect) $+\mathrm{SS}$ (error)]. The main effect of visual similarity was significant $[F(1,15)=105.80$, $\left.p<.01, \eta^{2}=.88\right]$. As shown in Fig. 4, detection sensitivity $d^{\prime}$ was significantly lower when the pre- and post-change targets were visually similar $(1.68 \pm .09)$ than when the two were dissimilar $(2.22 \pm .10)$. The main effect of conceptual similarity was also significant $[F(1,15)=51.31$,

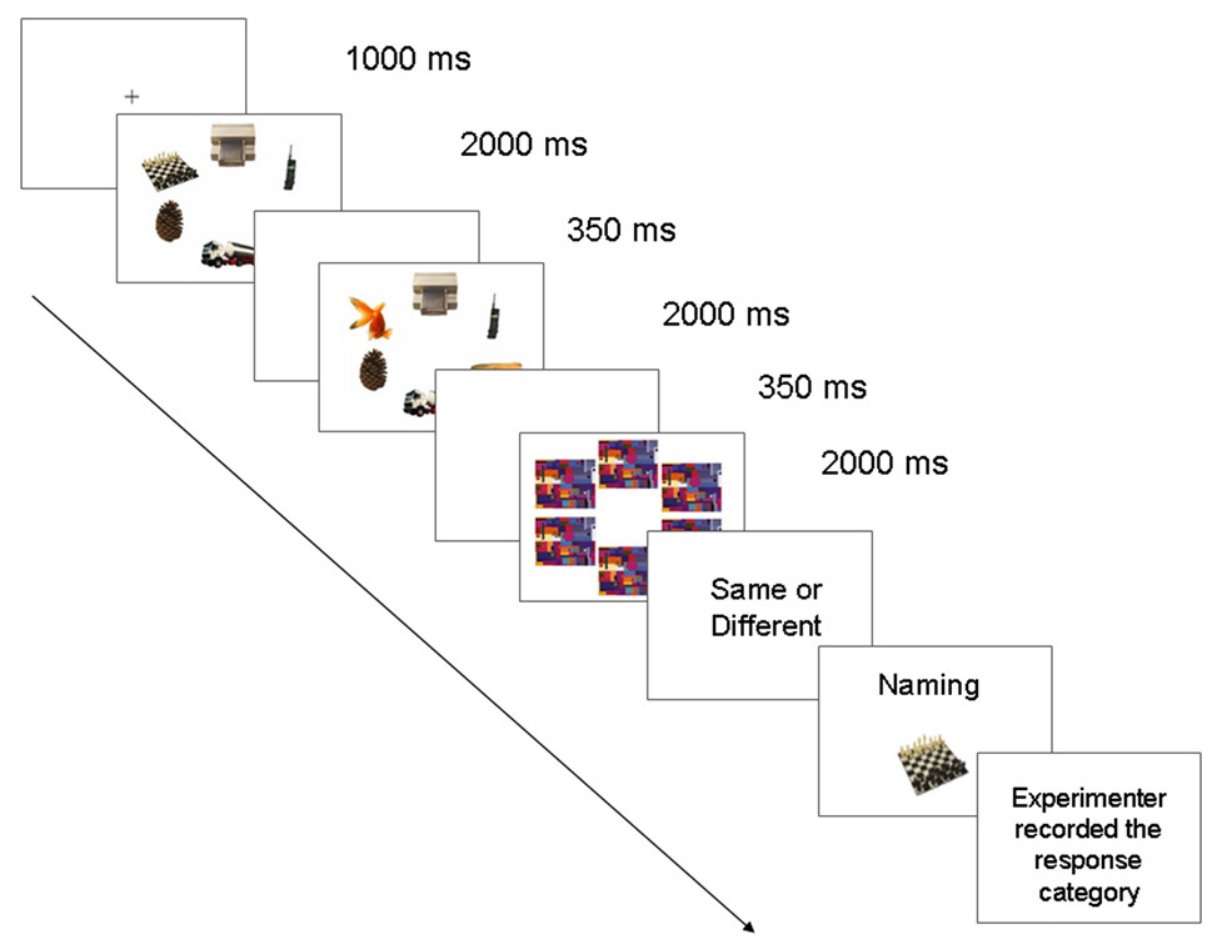

Fig. 2. The trial procedure used in Experiment 1. A change detection task was performed prior to object identification. 


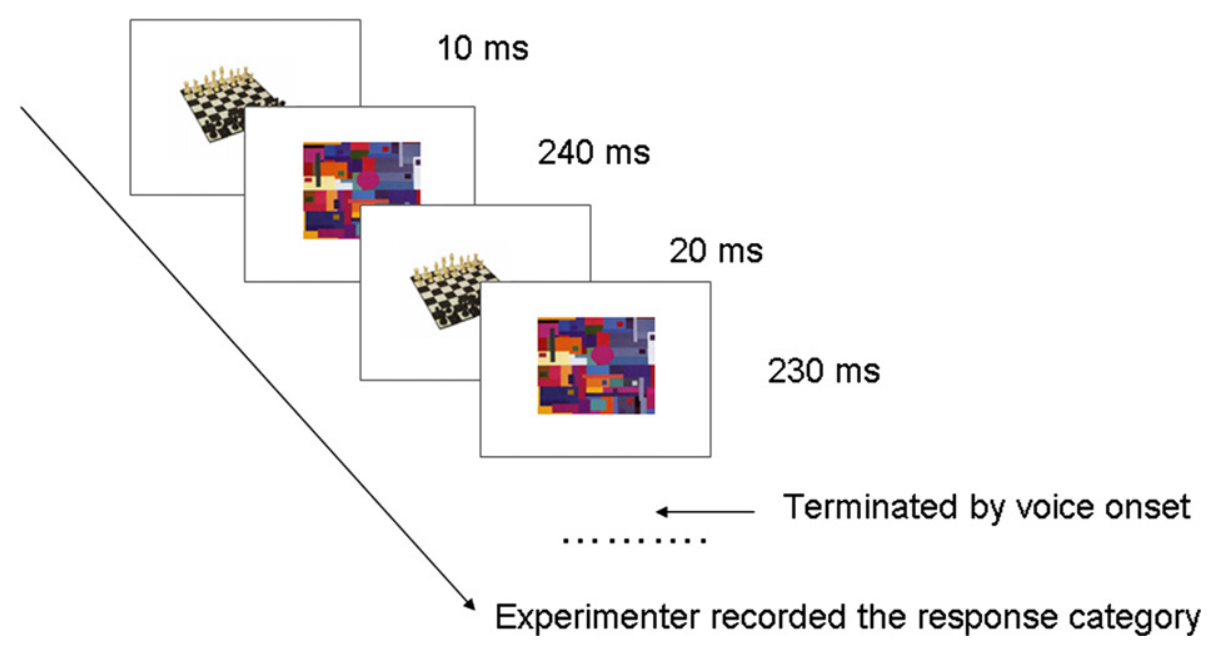

Fig. 3. A schematic diagram illustrates the procedure of the continuous identification task used in Experiment 1 . Stimulus duration gradually increased until participants named the object.

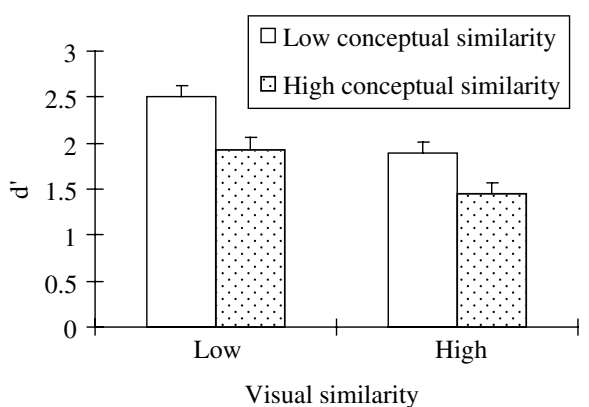

Fig. 4. Mean detection sensitivity $d^{\prime}(+\mathrm{SE})$ as a function of visual and conceptual similarities in Experiment 1. The two similarity manipulations additively affected detection sensitivity.

$p<.01, \eta^{2}=.77$ ], with a lower sensitivity when the two were conceptually similar $(1.69 \pm .10)$ than when the two were dissimilar $(2.21 \pm .09)$. The interaction effect was marginal $(p<.1)$, with the similarity on one dimension showing a smaller effect when the two targets already shared similarity on the other dimension than when the two targets did not share similarity on the other dimension.

\subsubsection{Object identification}

Detection failure only included trials in which participants missed a change. Two separate 2 (visual similarity) $\times 2$ (conceptual similarity) $\times 2$ (test object: new $/$ pre-change target) repeated measures ANOVAs were conducted on median reaction time (RT) for both correct change detection and detection failure. Using the criterion of having at least five observations in every cell of the design, data from six participants were included under detection failure and from nine participants under correct detection. We focused on the repetition priming effect.

Analysis of the accuracy data showed null effects $(p s>.1)$. Participants were quite accurate at object identification under detection failure $.95 \pm .01$ and $.96 \pm .01$ for the pre-change target and the new object, respectively) and under correct detection $(.94 \pm .01$ and $.94 \pm .01$ for the pre-change target and the new object, respectively). Repetition priming was significant in RT under detection failure $\left[F(1,5)=9.27, p<.05, \eta^{2}=.67\right]$, with a faster identification time for naming the pre-change target $(1398.56 \pm 55.21 \mathrm{~ms})$ than for naming the new object $(1537.23 \pm 57.57 \mathrm{~ms})$. Repetition priming was also significant under correct detection $[F(1,8)=14.69, p<.01$, $\left.\eta^{2}=.65\right]$, with a faster identification time for naming the pre-change target $(1397.45 \pm 49.07 \mathrm{~ms})$ than for naming the new object $(1580.07 \pm 61.40 \mathrm{~ms})$. Similarity manipulations did not affect identification of the pre-change target under detection failure or under correct detection $(p s>.3)$. Fig. 5 presents the overall effect.

We also pooled raw data across similarity manipulations to include all participants in a non-parametric bootstrapping method to fit each individual participant's reaction time distribution with an ex-Gaussian function because RT distributions are generally positively skewed. The exGaussian probability density function is specified as follows:

$f(x)=\frac{1}{\tau} \exp \left(\frac{\mu}{\tau}+\frac{\sigma^{2}}{2 \tau^{2}}-\frac{x}{\tau}\right) \Phi\left(\frac{x-\mu-\sigma^{2} / \tau}{\sigma}\right)$

The parameter $\mu$ reflects the mean of the normal distribution in the function, the parameter $\sigma$ indicates the standard deviation of the normal distribution, and the parameter $\tau$ measures the exponential tail that represents the skew of the RT distribution. We conducted a 2 (detection accuracy: incorrect/correct) $\times 2$ (test object) repeated measures ANOVA on each parameter. The analysis of the parameter $\mu$ (Fig. 5c) showed a marginal effect of detection accuracy $(p<.1)$, with a trend of faster RT in object identification after correct detection. The main effect of test object was significant $\left[F(1,15)=5.89, p<.05, \eta^{2}=.28\right]$, with a smaller mean for identifying the pre-change target $(876.29 \pm 22.57 \mathrm{~ms})$ than for identifying the new object $(966.95 \pm 42.85 \mathrm{~ms})$. The interaction was not significant 

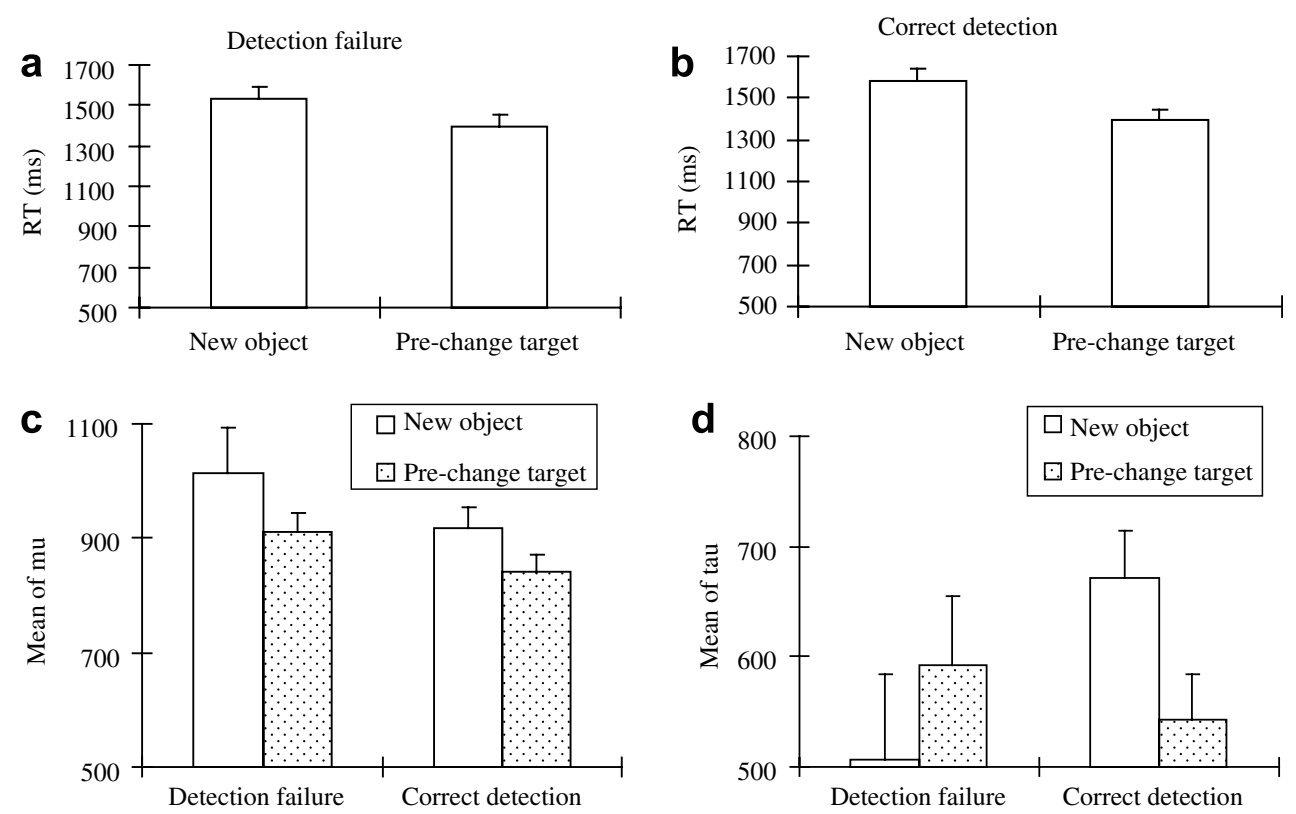

Fig. 5. Identification of the pre-change target and the new object under (a) detection failure $(n=6)$, and (b) correct detection ( $n=9)$. (c) Averaged $\mu$ of the ex-Gaussian function as a function of detection accuracy $(n=16)$. (d) Averaged $\tau$ of the ex-Gaussian function as a function of detection accuracy ( $n=16$ ). Repetition priming was observed in (a)-(c), and the exponential tail in the RT distribution for naming the new object was longer than the tail for naming the pre-change target under correct detection.

$(p>$.7). Analysis of the parameter $\sigma$ showed a marginal effect of test object $(p<.1)$, with a slightly larger variability when identifying the new object. The analysis of parameter $\tau$ (Fig. 5d) showed a significant interaction $[F(1,15)=6.49$, $\left.p<.05, \eta^{2}=.30\right]$. The mean of the exponential tail was significantly larger for identifying the new object under correct detection $\left[F(1,30)=7.56, p<.05, \eta^{2}=.20\right]$, but not for identifying the pre-change target $(p>.4)$.

The analysis of the RT distribution for naming objects based on all participants' data supports the findings based on a small sample derived by the use of five observations as a criterion for excluding participants from further analysis. The mean of the normal distribution showed the repetition priming effect regardless of detection accuracy. There were more extreme values in the RT distribution when naming the new object after correct detection. During identification, participants might at times have retrieved and compared the new object with target representations after they consciously detected a change and were aware of the two targets. As a result, extreme values in the RT distribution were related to identifying the new object.

\subsection{Discussion}

The results showed that both visual and conceptual similarities lowered detection sensitivity. Similarity did not matter for identifying the pre-change object under detection failure. The results are in accord with the prediction of the comparison failure (Mitroff et al., 2004) or retrieval failure (Hollingworth, 2003) hypothesis. Reaction time for naming the pre-change target was significantly faster than for naming the new object under both detection failure and correct detection. The pre-change target was also identified at about the same speed under both conditions. The level of the pre-change representation under detection failure was equivalent to that under correct detection.

Although our results replicate Silverman and Mack's (2006) findings under detection failure, the repetition priming effect under correct detection does not support their inhibition/attenuation hypothesis. Methodological differences may have caused the inconsistent findings. The stimuli were presented for $100 \mathrm{~ms}$ in their study and $2000 \mathrm{~ms}$ in this experiment. In their study, the post-change display was not followed by a mask and their participants were required to report the row where a change occurred before performing the identification task. This experimental context may have encouraged memory retrieval of the postchange array so that the post-change letters had a higher activation. We used a mask after the post-change display and our participants were not required to report where a change had occurred before performing the identification task. Repetition priming was observed for identifying the pre-change target under correct detection. Future research should investigate how these methodological differences may influence the repetition priming effect under detection failure and under correct detection.

\section{Experiment 2}

The objective of this experiment was to investigate how similarity can affect explicit recognition of the pre-change object. Dissociation between change detection and memory recognition as a function of similarity was predicted. We tested this prediction with a $2 \mathrm{AFC}$ recognition test to 
demonstrate that object recognition and change detection rely on different types of information despite overlapping processes, and algorithms.

\subsection{Method}

\subsubsection{Participants}

Twenty one undergraduate students volunteered for this experiment to receive a bonus credit in an introductory psychology course. All participants were aged 18-24 and had normal or corrected-to-normal vision.

\subsubsection{Stimuli, design, and procedure}

The same stimuli and design as in Experiment 1 were used except for the following two modifications. First, the total number of trials was reduced to 180 , with the ratio for trial composition remaining the same as in Experiment 1. Second, two 2AFC recognition tests were performed after change detection on the pre-change target (pre2AFC) and the post-change target (post-2AFC). The test order was randomized across trials. A new object that had not occurred in either stimulus array was used as a lure in each recognition test. The test target was positioned equally often on the right or left side of the screen center. Participants pressed the left or right mouse button to indicate the target location.

\subsection{Results}

\subsubsection{Change detection}

The results from a 2 (visual similarity) $\times 2$ (conceptual similarity) repeated measures ANOVA showed a significant effect of visual similarity on the $d^{\prime}$ data $\left[F(1,20)=76.18, p<.01, \eta^{2}=.79\right]$. As shown in Fig. 6, sensitivity in change detection between two visually similar targets was significantly lower $(1.36 \pm .07)$ than with two dissimilar targets $(2.05 \pm .09)$. No other effects reached significance $(p \mathrm{~s}>.7)$. Visual similarity decreased detection sensitivity.

\subsubsection{Recognition of the pre-change target under detection failure}

We used a criterion of having at least three observations in every cell to exclude participants from further data anal-

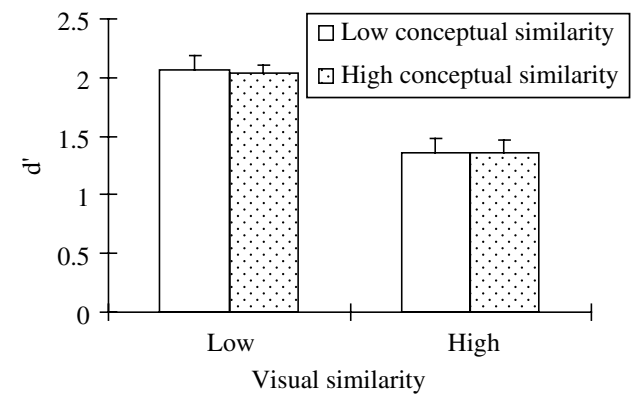

Fig. 6. Detection sensitivity $d^{\prime}$ as a function of visual and conceptual similarity in Experiment 2. Visual similarity degraded sensitivity. ysis. Thirteen participants' data were analyzed under detection failure. The results from a 2 (visual similarity) $\times 2$ (conceptual similarity) repeated measures ANOVA on pre-change recognition showed that the main effect of visual similarity was significant $[F(1,12)=5.43, p<.05$, $\left.\eta^{2}=.31\right]$ and the main effect of conceptual similarity was also significant $\left[F(1,12)=17.81, p<.01, \eta^{2}=.60\right]$. The interaction was not significant $(p>.3)$. Recognition of the pre-change target (Fig. 7) was more accurate when the two targets were visually similar $(.73 \pm .04)$ than when the targets were dissimilar $(.64 \pm .04)$. Pre-change recognition was more accurate when the two targets were conceptually similar $(.77 \pm .03)$ than when the targets were dissimilar $(.60 \pm .04)$. Similarity improved recognition.

\subsubsection{Recognition of the pre-change target under correct detection}

All participants' data were analyzed under correct detection. The results from a 2 (visual similarity) $\times 2$ (conceptual similarity) repeated measures ANOVA showed that prechange recognition was significantly affected by visual similarity $\left[F(1,20)=14.07, p<.01, \eta^{2}=.41\right]$. The two similarity manipulations also significantly interacted $\left[F(1,20)=11.66, p<.01, \eta^{2}=.37\right]$. As shown in Fig. 8, conceptual similarity was significant only when the two targets were visually dissimilar $[F(1,40)=13.12, p<.01$, $\left.\eta^{2}=.25\right]$ and the effect was not significant when the two targets were visually similar $(p>.1)$. Visual similarity was

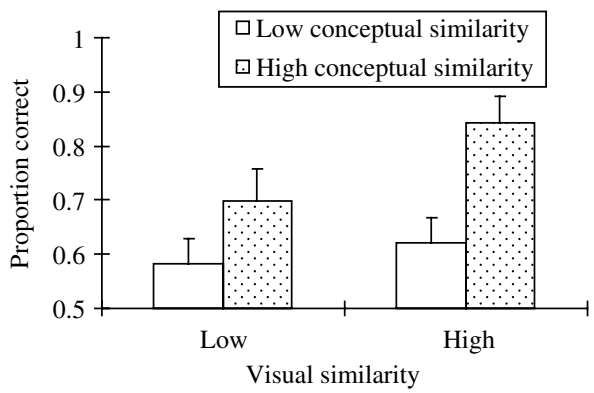

Fig. 7. Recognition of the pre-change target under detection failure as a function of visual and conceptual similarity in Experiment $2(n=13)$. The two similarity manipulations additively improved pre-change recognition.

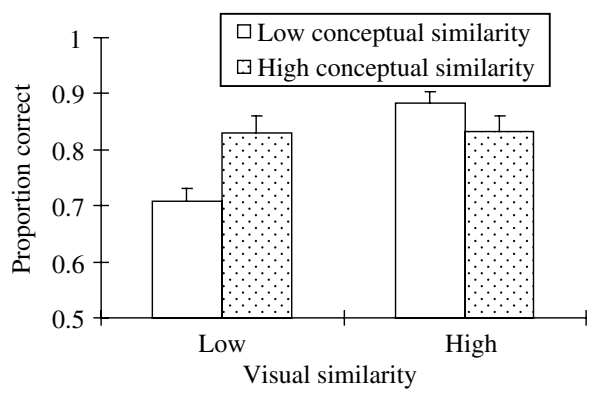

Fig. 8. Recognition of the pre-change target under correct detection as a function of visual and conceptual similarities in Experiment $2(n=21)$. Pre-change recognition was better when the two targets shared similarity on one dimension. 
significant only when the two targets were conceptually dissimilar $\left[F(1,40)=25.58, p<.01, \eta^{2}=.39\right]$ and not when the two targets were conceptually similar $(p>9)$. Similarity on one dimension was sufficient to enhance recognition.

\subsubsection{Contrasting recognition performance under detection failure and correct detection}

We also conducted a 2 (detection accuracy) $\times 2$ (target type: pre-change, post-change) $\times 2$ (visual similarity) $\times 2$ (conceptual similarity) repeated measures ANOVA to investigate memory performance with 13 participants' data. The main effect of detection accuracy was significant $\left[F(1,12)=21.33, p<.01, \eta^{2}=.64\right]$, with better performance under correct detection $(.85 \pm .01)$ than under detection failure $(.73 \pm .01)$. Detection accuracy did not interact with target type $(p>.6)$ and none of the higherorder interaction effects involving these two variables were significant $(p s>.1)$. As shown in Fig. 9, the three-way interaction among target type, visual similarity, and conceptual similarity was significant $[F(1,12)=5.86, p<.05$, $\left.\eta^{2}=.33\right]$. The contrast between pre-change and postchange recognition was significant in all conditions except when the two targets were visually and conceptually similar.

\subsection{Discussion}

The results showed that visual similarity lowered detection sensitivity, yet both visual and conceptual similarities improved recognition of the pre-change target. Dissociation arose as predicted. In addition to the dissociation effect, the analysis of memory performance under detection failure and correct detection showed that recognition performance was significantly better under correct detection than under detection failure. More important, this effect did not interact with target type. Memory performance was better under correct detection for both the pre- and post-change targets. The degree of explicit access to memory representation was lower under detection failure.

When explicit access was weak under detection failure, visual and conceptual similarity additively improved

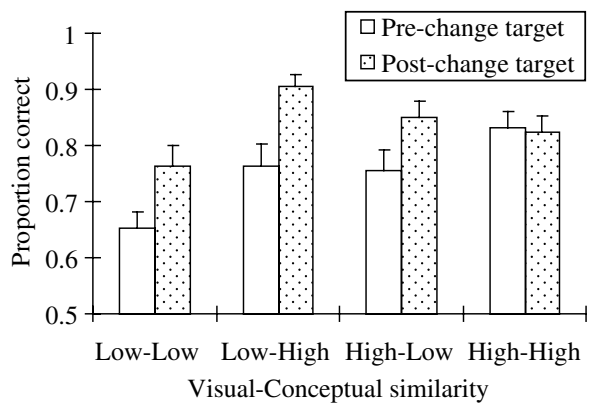

Fig. 9. Recognition of the pre-change target under both detection failure and correct detection as a function of target type and similarity manipulations in Experiment $2(n=13)$. Pre-change recognition was worse than post-change recognition unless the two targets shared both visual and conceptual similarities. pre-change recognition to a level equivalent to post-change recognition. When a pre-change representation could be explicitly accessed for correct detection, the two similarity manipulations interacted in affecting recognition. Adding a second dimension of similarity provided little utility under correct detection if the two targets shared one type of similarity. Similarity increases the likelihood that a pre-change target can be recognized. The difference in the degree of explicit access between detection failure and correct detection modulates how the two similarity mechanisms support the incremental benefit in recognition.

\section{Experiment 3}

The objective of this experiment was to investigate the similarity effect on the recognition of a pre-change object with incomplete representation. It is plausible that the representation of a pre-change target is volatile as the overwriting hypothesis suggests (Beck \& Levin, 2003; see Simons, 2000). Yet, an incomplete representation can support the repetition priming effect observed in Experiment 1 and the similarity effect observed in Experiment 2. It is critical to examine whether an incomplete representation can lead to these results.

We used the similarity effect to test whether the similarity benefit on pre-change recognition can arise from using a similar post-change target to retrieve an incomplete prechange representation or from matching a test probe to memory traces that contain an incomplete pre-change representation. To emulate an incomplete representation, we superimposed a random-dot mask with $20 \%$ dot density on each pre-change stimulus. Such masking should reduce visual features so that visual representations do not have all the details and conceptual information may not be encoded.

None of the stimuli were masked in Experiment 2 and the results showed a similarity effect. We interpreted this finding as resulting from increasing the match signals by similarity in the test probe. In this experiment, we masked only the pre-change stimuli and kept the post-change stimuli and the test probes intact. Stimulus degradation leads to less elaborate proximal stimulus representation in perception and memory (Op de Beeck, Béatse, Wagemans, Sunaert, \& Van Hecke, 2000; Op de Beeck \& Wagemans, 2001). If a test probe can rely on a similar post-change target to retrieve an incomplete pre-change representation, we expected to observe a similarity benefit in this experiment. If the similarity effect observed in Experiment 2 arose from matching an intact test probe to the memory traces that contained an incomplete pre-change target representation, the similarity effect should also be observed in this experiment.

Snodgrass and Hirshman (1994) showed that a match between study and test line-drawing objects affects recognition. Recognition was more accurate when the two stimuli matched in the degree of fragmentation than when the two were mismatched. Superimposing a $20 \%$ mask on the 
pre-change object may have two consequences. First, similarity based on an incomplete pre-change representation and an intact post-change target is reduced. Second, similarity based on an incomplete pre-change representation and an intact test probe is reduced. The similarity effect observed in Experiment 2 may be reduced or eliminated in this experiment. If this is the case, the results would suggest that the similarity effect observed in Experiment 2 arose from retrieving or matching a pre-change representation that was almost complete.

\subsection{Method}

\subsubsection{Participants}

Eighteen undergraduates volunteered for this experiment to receive a bonus credit for an introductory psychology course. All participants were aged 18-22 and had normal or corrected-to-normal vision.

\subsubsection{Stimuli, design, and procedure}

The same stimuli and design as in Experiment 2 were used in this experiment except that masked pre-change stimuli were used. A random-dot mask was superimposed on each pre-change stimulus with $20 \%$ dot density. Each dot was a square of $4 \times 4$ pixels. The level of dot density was defined by the percentage of a $120 \times 120$ pixel object image covered by the superimposed mask of dots. Fig. 10 shows examples of masked pre-change and unmasked post-change target pairs in each of the similarity conditions. As shown in this figure, the masked stimuli are identifiable.

\subsection{Results}

\subsubsection{Change detection}

The results from a 2 (visual similarity) $\times 2$ (conceptual similarity) repeated measures ANOVA on detection sensitivity showed a significant main effect of visual similarity
$\left[F(1,17)=36.26, p<.01, \eta^{2}=.38\right]$ and no other effect was significant $(p s>.3)$. As shown in Fig. 11, detection sensitivity was higher $(1.12 \pm .09)$ when the two targets were visually dissimilar than when the two targets were similar $(.65 \pm .07)$. Visual similarity degraded detection performance.

\subsubsection{Recognition of the pre-change target under detection failure}

Three participants were quite accurate in change detection and their data were excluded from further analysis according to the criterion used in Experiment 2. Analysis of the 15 participants' data showed no significant results ( $p s>.1$ ), as Fig. 12 shows.

\subsubsection{Recognition of the pre-change target under correct detection}

All participants' data were analyzed and the results showed a significant main effect of conceptual similarity $\left[F(1,17)=7.09, p<.05, \eta^{2}=.29\right]$, with a better performance when the two targets were conceptually similar $(.80 \pm .02)$ than when the targets were dissimilar $(.73 \pm .02)$. No other effects were significant $(p s>.1)$. Fig. 13 shows the results.

\subsubsection{Contrasting recognition performance under detection failure and correct detection}

The results from a 2 (detection accuracy) $\times 2$ (target type) $\times 2$ (visual similarity) $\times 2$ (conceptual similarity) repeated measures ANOVA on the 15 participants' data showed that the main effect of detection accuracy was significant $\left[F(1,14)=43.69, p<.01, \eta^{2}=.76\right]$. Recognition performance was better under correct detection $(.82 \pm .01)$ than under detection failure $(.70 \pm .02)$. The main effect of target type was also significant $\left[F(1,14)=46.99, p<.01, \eta^{2}=.77\right]$, with post-change recognition $(.82 \pm .01)$ being better than pre-change recognition $(.70 \pm .02)$. Detection accuracy did not interact with

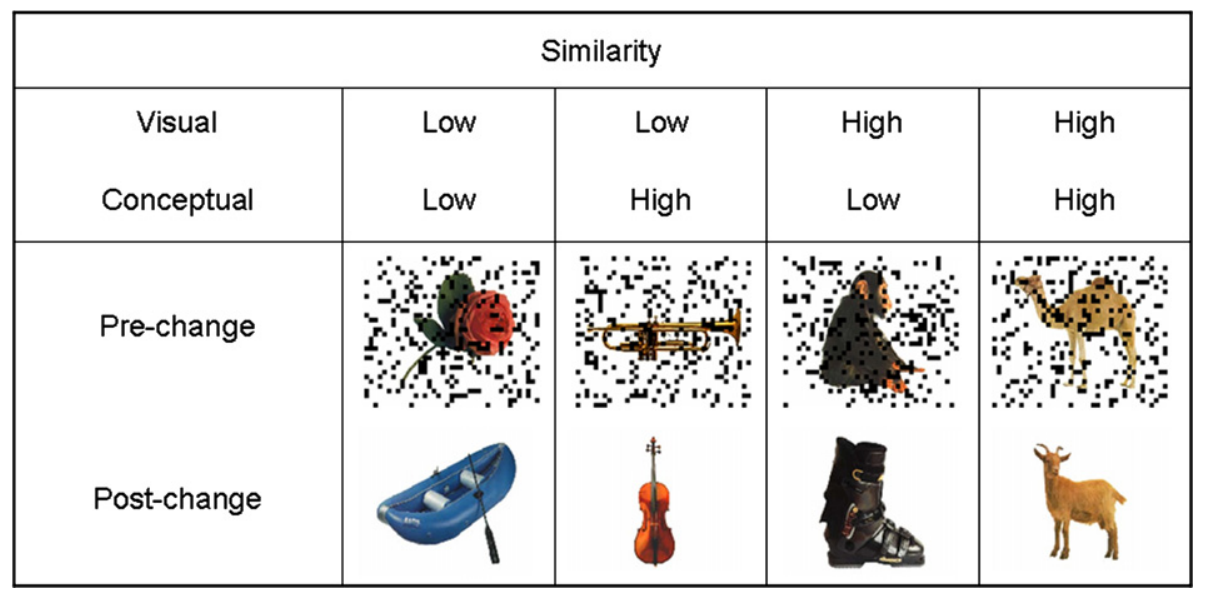

Fig. 10. An example of masked pre-change targets and intact post-change targets in the four conditions used in Experiment 3 . A masked stimulus can still be identified. 


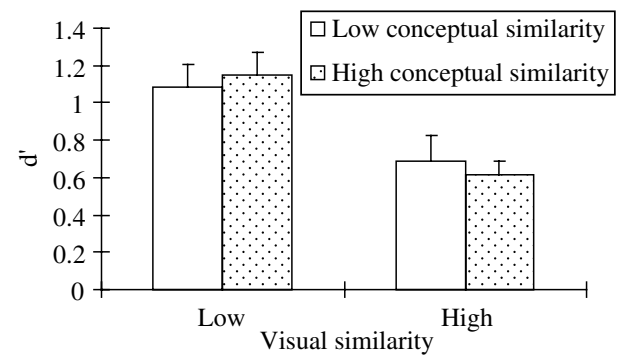

Fig. 11. Detection sensitivity $d^{\prime}$ as a function of visual and conceptual similarity in Experiment 3. Visual similarities degraded sensitivity.

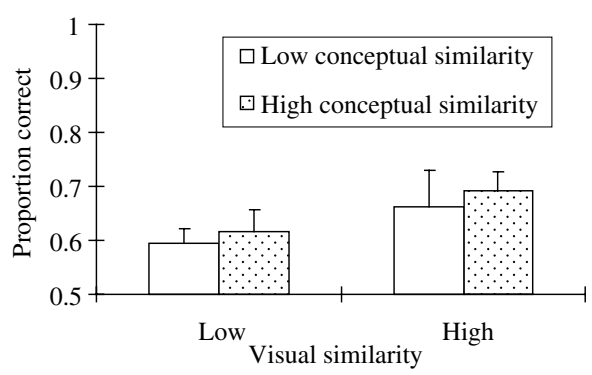

Fig. 12. Recognition of the pre-change target under detection failure as a function of visual and conceptual similarities in Experiment $3(n=15)$. No significant results were observed.

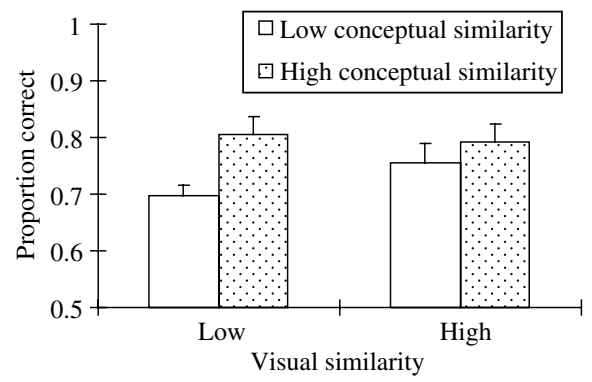

Fig. 13. Recognition of the pre-change target under correct detection as a function of visual and conceptual similarities in Experiment $3(n=18)$. Conceptual similarity improved recognition.

target type $(p>.8)$ and none of the higher-order interactions involving these two variables were significant $(p \mathrm{~s}>.2)$.

\subsubsection{Contrasting detection performance as a function of masking}

We conducted a 2 (experiment $) \times 2$ (visual similarity $) \times 2$ (conceptual similarity) mixed design ANOVA on detection sensitivity. The main effect of experiment was significant $\left[F(1,37)=40.47, p<.01, \eta^{2}=.52\right]$, with higher sensitivity in Experiment 2 without masking $(1.70 \pm .07)$ than in Experiment 3 with masking $(.88 \pm .06)$. The contrasts showed results consistent with the findings in the analysis of each experiment, with a significant visual similarity effect and null conceptual similarity effect in both experiments.

\subsubsection{Contrasting pre-change recognition under detection failure as a function of masking}

The results showed that the main effect of the experiment was not significant $(p>.2)$ and the contrasts showed results consistent with the findings in the analysis of each experiment with significant visual and conceptual similarity effects in Experiment 2 without masking. No significant similarity effect was observed in Experiment 3 with masking.

The contrast of pre-change recognition performance without and with masking showed that performance under detection failure was not significantly different between the two experiments except when the two targets were highly similar. Pre-change recognition in Experiment 2 was significantly better than recognition in Experiment 3, $t(26)=2.57, p<.05$ when the two targets were visually and conceptually similar.

\subsection{Discussion}

The results showed that visual similarity degraded change detection performance as observed in Experiment 2 . With $20 \%$ of visual information masked, detection sensitivity was lower than when it was based on unmasked prechange stimuli in Experiment 2. The amount of visual information influences change detection. Memory performance was better under correct detection than under detection failure, replicating the results of Experiment 2. Explicit accessibility of memory representations was lower under detection failure for both the pre- and post-change targets.

When the explicit accessibility was low under detection failure, the contrast between Experiments 2 and 3 showed that similarity benefits were much reduced with masked stimuli. We used the formula (at an alpha level of .05 and statistical power of .80) specified for a randomized block design (Kirk, 1995) to estimate the sample size that would be required for each similarity effect to become significant under detection failure in Experiment 3. A sample of 344 and 1088 participants would be required for the visual similarity effect and conceptual similarity effect, respectively. In contrast, a sample of 13 participants showed significant visual and conceptual similarity benefits under detection failure in Experiment 2. The relative difficulty in obtaining similarity benefits under detection failure in this experiment is of importance given that only $20 \%$ of visual information is masked and the post-change stimuli and test probes are all intact.

The relative difficulty of observing similarity benefits under detection failure in this experiment leads to two findings. First, pre-change recognition in Experiment 2 was significantly better than recognition in Experiment 3 only when the two targets were highly similar. Second, prechange recognition in Experiment 2 was not worse than post-change recognition when the two targets were highly similar. In contrast, pre-change recognition in Experiment 3 was significantly worse than post-change recognition even when the two targets were highly similar. 
When participants could explicitly access the masked pre-change representation for correct detection, it appears that they could label the object at the semantic level so that they could utilize conceptual similarity to improve prechange recognition under correct detection. Participants did not utilize the incomplete visual information in recognition under correct detection.

\section{General discussion}

This study investigated how a similar post-change object affects memory of the pre-change object and change detection. Similarity did not influence the pre-change representation as shown with an object identification task in Experiment 1. Repetition priming was observed for identifying the pre-change object under both detection failure and correct detection, at a faster speed than identifying the new object. Similarity improved recognition of the pre-change object in Experiment 2 when the pre-change stimuli were unmasked. The similarity benefit to recognition was reduced when pre-change stimuli were masked in Experiment 3 so that no benefit was found for recognition under detection failure and only the conceptual similarity benefit was observed under correct detection. In all three experiments, similarity deteriorated performance in change detection.

The results taken together suggest that the level of prechange representation under detection failure is equivalent to the level of representation under correct detection and the pre-change representation is almost complete in the present experimental context. Despite the almost complete representation, the pre- and post-change objects may be inaccessible for correct detection, as recognition was worse under detection failure than under correct detection regardless of target type in both Experiments 2 and 3. When the pre- and post-change objects were accessed for detection, similarity increased comparison difficulty and detection sensitivity decreased. Memory recognition and change detection are dissociable as they rely on different types of information in the decision process. Recognition of a pre-change object relies on the match-to-mismatch signals when comparing a test probe to memory traces. Similarity increases the match signals. Change detection relies on the mismatch-to-match signals when comparing the pre- and post-change objects. Similarity decreases the mismatch signals.

\subsection{Object representations under detection failure and under correct detection}

Although previous studies have demonstrated the existence of visual representation under change blindness (Hollingworth et al., 2001; Mitroff et al., 2004; Ryan \& Cohen, 2004; Silverman \& Mack, 2006; Simons et al., 2002), the completeness of the pre-change representation was unresolved given that a partial representation can be recalled with appropriate cues, can aid recognition, can bias eye movement, and can lead to repetition priming. The repetition priming effect observed in Experiment 1 does not necessarily reflect the completeness of the pre-change representation. The contrast between Experiments 2 and 3 in the similarity effect provides the critical evidence that an almost complete representation exists under detection failure and under correct detection. More than $80 \%$ of the visual information is required in the pre-change representation to observe a similarity benefit under detection failure.

The occurrence of a post-change object does not overwrite the pre-change object as the overwriting hypothesis (Beck \& Levin, 2003; see Simons, 2000) suggested. According to the overwriting hypothesis, the post-change object overwrites the pre-change object representation so that the latter becomes volatile. If this is the case, similarity should dampen both detection performance and memory recognition as the magnitude of backward masking is accentuated by the similarity between a target and the subsequent mask (see Enns \& Di Lollo, 2000) and similar stimuli can retroactively reduce the discriminability of previous signals in visual short-term memory (Lakha \& Wright, 2004). Yet, repetition priming was observed under detection failure and similarity led to a benefit rather than a cost to recognition performance. Moreover, the repetition priming effect under detection failure was found to be equivalent to the priming effect under correct detection in Experiment 1.

The results show that the pre-change object representation is not inhibited or attenuated for correct detection as Silverman and Mack (2006) suggested. Silverman and Mack (2006) suggested that the pre-change stimulus representation is inhibited or attenuated to guard the change information for correct detection. Yet, detection accuracy did not alter the repetition priming effect in Experiment 1. This finding was observed in the analysis based on the reaction time from a small sample of participants and also in the analysis based on the parameters fitted for each individual participant's reaction time distribution. It is uncertain what has caused the inconsistent findings between the present study and Silverman and Mack's (2006) study. Further exploration is required to understand which aspects of the methodological differences lead to the inconsistent results.

The nature of visual representations has been investigated with different methodologies. Becker and Pashler (2005) argued that the change detection paradigm may not be ideal to investigate the amount of information that is acquired while viewing a visual scene. They used a memory paradigm to show that a limited number of three stimuli can be encoded in visual memory while viewing a visual scene of letters. Using a direct test of memory, VanRullen and Koch (2003) showed that six objects can be reliably retained from a single fixation and four can be consolidated into visual short-term memory. Using a change detection paradigm, Hollingworth (2004) suggested that on-line visual representations contain two objects from visual 
short-term memory and also a detailed representation retrieved from visual long-term memory. Length of viewing time may play a role in the amount of information retained, as Liu and Jiang (2005) demonstrated that only 1 of 10 objects can be retained from a single glimpse with a short viewing time of $250 \mathrm{~ms}$ and five objects can be remembered when the viewing time is self-paced. Without any scene context, the results of this study suggest that six objects are retained with almost complete representations in visual short-term memory after viewing these objects for $2000 \mathrm{~ms}$.

\subsection{Explicit access to memory representation in the context of change detection}

Despite being almost completely stored in memory, these representations may not be explicitly accessed during change detection. The results of both Experiments 2 and 3 reveal that recognition performance is lower under detection failure than under correct detection regardless of target type. Given that the level of representation under detection failure is equivalent to that under correct detection, it appears that the inability to explicitly access the pre- and post-change objects is a reason for change detection to fail.

It is still unknown what causes memory representation to be explicitly inaccessible for conscious change detection. Many accounts have been proposed, including disconnection with consciousness (Ryan \& Cohen, 2004), lack of attentional focus (Enns \& Di Lollo, 2000), and retrieval failure (Hollingworth, 2003). Whether retrieval cues match the cues used in stimulus encoding may also play an important role (Zeelenberg, 2005). All these factors can cause memory representations to be explicitly inaccessible.

In addition to explicit inaccessibility, similarity between the pre- and post-change targets also led to detection failure. Nevertheless, similarity between the two targets enhanced the accessibility of the pre-change target under detection failure to the degree that pre-change recognition was as good as post-change recognition when the prechange representation was almost complete. As predicted by global memory models (Hintzman, 1988; Murdock, 1997; Shiffrin \& Steyvers, 1997), similarity benefits arose from increasing the match signal in memory probing. Visual similarity and conceptual similarity additively improved pre-change recognition under detection failure as shown in Experiment 2.

When the pre-change representation was incomplete, the similarity benefits under detection failure were greatly reduced. In contrast with the results in Experiment 2, the conceptual similarity effect was reduced to a greater extent than the visual similarity effect under detection failure in Experiment 3. Participants could not process the masked pre-change representation to the conceptual level when they could not access the representation. Without explicit knowledge of object semantics, participants utilized the match signal on the incomplete visual information in memory representation during recognition.

When the pre- and post-change objects were accessed and compared to support successful change detection, the degree of memory access was greater than that under detection failure, and recognition performance was better under correct detection. As the pre-change object was already accessed, the marginal utility of adding similarity on a second dimension was small and the two similarity manipulations interactively influenced pre-change recognition when the representation was almost complete in Experiment 2. When the representation was incomplete as in Experiment 3 , only conceptual similarity improved pre-change recognition as the participants were aware of the fact that the visual information in the explicitly accessed representation was incomplete.

As detection failure can arise from the failure to access both the pre- and post-change objects (access failure) or from the failure to detect differences between similar objects after successful access (comparison failure), whether similarity increases explicit access to the ones in the former, the latter, or both contexts is an issue for future research to explore. Two lines of evidence tentatively suggest that similarity benefits are not limited to reinstating the successfully accessed representation. The failure rate of change detection was lower in Experiment 2 than in Experiment 3 , with a sensitivity level in the former twice the latter. Yet, the overall recognition performance under detection failure was not significantly different between the two experiments. This contrast is inconsistent with the suggestion that similarity improves recognition of an accessed representation only, unless complicated assumptions about the ratio of access failure to comparison failure in each experiment are added to the explanation. Without the assumptions of unequal ratios, the finding that similarity benefits are observed in Experiment 2 and not in Experiment 3 under detection failure is also inconsistent with the suggestion that similarity improves recognition of the accessed representation only. The correlation between brain activity during change detection and later recognition performance should provide further evidence to resolve the issue.

\subsection{Change blindness}

The results from the three experiments suggest that three factors influence the accuracy of change detection: the level of stimulus encoding, the degree of explicit access to memory representations of the pre-change and post-change targets, and the similarity between the two targets. These findings are consistent with the results observed in previous studies of change blindness.

The contrast in detection sensitivity between Experiments 2 and 3 highlights that the amount of visual information is critical for change detection. Detection sensitivity was higher with the unmasked pre-change display in Experiment 2 than with the masked pre-change display in Experiment 3. The impact of the encoding level on change 
blindness has been demonstrated in previous studies. The use of mud splashes (O'Regan, Rensink, \& Clark, 1999) is an example that blocking stimulus encoding can lead to change blindness. The use of spatial cueing to focus attention on the to-be-changed location (Landman, Spekreijse, \& Lamme, 2003; Schmidt, Vogel, Woodman, \& Luck, 2002) is another example that encoding under focused attention can improve change detection. The pattern of event-related potentials in the encoding of the pre-change display also predicts change detection performance (Koivisto \& Revonsuo, 2005).

The finding that explicit access to memory representations is low under detection failure suggests that explicit inaccessibility of memory representations can lead to change blindness. This result is consistent with the suggestion that a reason for change blindness to occur is that the pre-change and post-change targets are not compared (Mitroff et al., 2004). Hollingworth (2003) also suggested that retrieval or comparison failure can lead to change blindness. Thus, failure to update the changed feature in memory (Droll, Hayhoe, Triesch, \& Sullivan, 2005) or focusing attention on a memory representation at a cued location where a change has recently occurred (Yeh, Yang, $\&$ Chiu, 2005) can influence detection accuracy.

The effect of visual similarity on detection accuracy has been demonstrated in previous studies (Mitroff et al., 2002; Smilek et al., 2000; Williams \& Simons, 2000; Zelinsky, 2003). This study also finds a reliable effect of visual similarity on detection sensitivity with a significant main effect across three experiments. The higher the visual similarity is, the lower the detection sensitivity would be. This finding supports the signal detection view of change detection (Wilken \& Ma, 2004). The effect of conceptual similarity is less reliable and was significant only in Experiment 1. The unreliable conceptual similarity effect may appear surprising as previous studies have shown that semantic categorization of natural scenes can be fast (Fabre-Thorpe et al., 2001). Studies using rapid stimulus presentation also found facilitation between semantically related pictures and words (O'Connor \& Potter, 2002) and perception of two natural scenes of the same category was better than perception of scenes of different categories (Evans \& Treisman, 2005).

It is likely that participants in Experiments 2 and 3 became consciously aware of the conceptual relatedness between the two targets in some trials during the recognition test. To guard change detection from being biased by conceptual relatedness, participants did not utilize conceptual information in judging whether there was a change between the two targets. Participants relied on the difference in visual properties to detect a change. In contrast, participants in Experiment 1 were not required to intentionally retrieve the pre-change and post-change targets. The participants in Experiment 1 may not have been consciously aware of the conceptual relatedness between targets. Conceptual information was hence utilized in computing the change magnitude for comparison.

\subsection{Object memory and change detection}

The encoding level, memory access, and decision difficulty affect detection accuracy at different stages. Visual masking affects the representation stored in visual memory, memory access and decision difficulty influence computation in comparison. The phenomenon of change blindness indeed does not indicate that detailed memory representations do not exist (see Simons \& Rensink, 2005 for discussion). Change detection and memory performance based on conscious access to representation may share many similar cognitive processes and may follow similar signal processing algorithms. They rely on different types of information. The relationship between object memory and change blindness may be more complex than what meets the eye.

\section{Acknowledgements}

This study was supported by grants (NSC94-2413-H002-017, NSC95-2413-H-002-003) from the National Science Council of Taiwan. Parts of this work were presented in November, 2005 at the 46th Annual Meeting of the Psychonomic Society, Toronto, Canada. We thank Ching-Fan $\mathrm{Chu}$ for her assistance in data collection.

\section{References}

Badgaiyan, R. D. (2000). Neuroanatomical organization of perceptual memory: an fMRI study of picture priming. Human Brain Mapping, 10, 197-203.

Battig, W. F., \& Montague, W. E. (1969). Category norms for verbal items in 56 categories: a replication and extension of the connecticut category norms. Journal of Experimental Psychology, 80, 1-46.

Beck, M. R., \& Levin, D. T. (2003). The role of representational volatility in recognizing pre- and post-change objects. Perception \& Psychophysics, 65, 458-468.

Becker, M. W., \& Pashler, H. (2005). Awareness of the continuously visible: information acquisition during preview. Perception \& Psychophysics, 67, 1391-1403.

Boucart, M., \& Humphreys, G. W. (1994). Attention to orientation, size, luminance, and color: attentional failure within the form domain. Journal of Experimental Psychology: Human Perception and Performance, 20, 61-80.

Coltheart, V. (1999). Introduction: perceiving and remembering brief visual stimuli. In V. Coltheart (Ed.), Fleeting memory: Cognition of brief visual stimuli (pp. 1-12). Cambridge, MA: MIT Press.

CorelDRAW! [Computer Software] (1994). Ottawa, ON, Canada: Corel, Inc.

Droll, J. A., Hayhoe, M. M., Triesch, J., \& Sullivan, B. T. (2005). Task demands control acquisition and storage of visual information. Journal of Experimental Psychology: Human Perception and Performance, 31, 1416-1438.

Dunn, J. C. (2004). Remember-know: a matter of confidence. Psychological Review, 111, 524-542.

Enns, J. T., \& Di Lollo, V. (2000). What's new in visual masking?. Trends in Cognitive Sciences 4, 345-352.

Evans, K., \& Treisman, A. (2005). Perception of objects in natural scenes: is it really attention free? Journal of Experimental Psychology: Human Perception and Performance, 31, 1476-1492.

Fabre-Thorpe, M., Delorme, A., Marlot, C., \& Thorpe, S. (2001). A limit to the speed of processing in ultra-rapid visual categorization of 
novel natural scenes. Journal of Cognitive Neuroscience, 13, $171-180$.

Gardiner, J. M., Konstantinou, I., Karayianni, I., \& Gregg, V. H. (2005). Memory awareness following speeded compared with unspeeded picture recognition. Experimental Psychology, 52, 140-149.

Hintzman, D. (1988). Judgments of frequency and recognition memory in a multiple trace memory model. Psychological Review, 95, 528-551.

Hirshman, E. (1998). On the utility of the signal detection model of the remember-know paradigm. Consciousness and Cognition, 7, 103-107.

Hollingworth, A. (2003). Failures of retrieval and comparison constrain change detection in natural scenes. Journal of Experimental Psychology: Human Perception and Performance, 29, 388-403.

Hollingworth, A. (2004). Constructing visual representations of natural scenes: the roles of short- and long-term visual memory. Journal of Experimental Psychology: Human Perception and Performance, 30, 519-537.

Hollingworth, A., \& Henderson, J. M. (1999). Object identification is isolated from scene semantic constraint: evidence from object type and token discrimination. Acta Psychologica, 102, 319-343.

Hollingworth, A., \& Henderson, J. M. (2002). Accurate visual memory for previously attended objects in natural scenes. Journal of Experimental Psychology: Human Perception and Performance, 28, 113-136.

Hollingworth, A., Williams, C. C., \& Henderson, J. M. (2001). To see and remember: visually specific information is retained in memory from previously attended objects in natural scenes. Psychonomic Bulletin \& Review, 8, 761-768.

Kirk, R. E. (1995). Experimental design: Procedures for the behavioral sciences (3rd ed.). Pacific Grove, CA: Brooks/Cole Publishing Company.

Koivisto, M., \& Revonsuo, A. (2005). Prechange event-related potentials predict change blindness in various attention conditions. Neuroreport, 16, 869-873.

Lakha, L., \& Wright, M. J. (2004). Capacity limitations of visual memory in two-interval comparison of Gabor arrays. Vision Research, 44, 1707-1716.

Landman, R., Spekreijse, H., \& Lamme, V. A. F. (2003). Large capacity storage of integrated objects before change blindness. Vision Research, 43, 149-164.

Li, F. F., VanRullen, R., Koch, C., \& Perona, P. (2002). Rapid natural scene categorization in the near absence of attention. Proceedings of the National Academy of Sciences of the United States of America, 99, 9596-9601.

Liu, K., \& Jiang, Y. (2005). Visual working memory for briefly presented scenes. Journal of Vision, 5, 650-658.

Mace, M. J., Thorpe, S. J., \& Fabre-Thorpe, M. (2005). Rapid categorization of achromatic natural scenes: how robust at very low contrasts? European Journal of Neuroscience, 21, 2007-2018.

Mitroff, S. R., Simons, D. J., \& Franconeri, S. L. (2002). The siren song of implicit change detection. Journal of Experimental Psychology: Human Perception and Performance, 28, 798-815.

Mitroff, S. R., Simons, D. J., \& Levin, D. T. (2004). Nothing compares 2 views: change blindness results from failures to compare retained information. Perception \& Psychophysics, 66, 1268-1281.

Murdock, B. B. (1997). Context and mediators in a theory of distributed associative memory (TODAM2). Psychological Review, 104, 839-862.

O'Connor, K. J., \& Potter, M. C. (2002). Constrained formation of object representations. Psychological Science, 13, 106-111.

Odegard, T. N., \& Lampinen, J. M. (2006). Memory editing: knowledge, criteria, and alignment. Memory, 14, 777-787.

Op de Beeck, H., Béatse, E., Wagemans, J., Sunaert, S., \& Van Hecke, P. (2000). The representation of shape in the context of visual object categorization tasks. Neuroimage, 12, 28-40.

Op de Beeck, H., \& Wagemans, J. (2001). Visual object categorisation at distinct levels of abstraction: a new stimulus set. Perception, 30, 1337-1361.

O’Regan, J. K., Rensink, R. A., \& Clark, J. J. (1999). Change-blindness as a result of "mudsplashes". Nature, $398,34$.
Parkhurst, D., Law, K., \& Neibur, E. (2002). Modeling the role of salience in the allocation of overt visual attention. Vision Research, 42, 107-123.

Pins, D., Meyer, M. E., Foucher, J., Humphreys, G., \& Boucart, M (2004). Neural correlates of implicit object identification. Neuropsychologia, 42, 1247-1259.

Rensink, R. A. (2000). Seeing, sensing, and scrutinizing. Vision Research, 40, 1469-1487.

Ryan, J. D., \& Cohen, N. J. (2004). The nature of change detection and online representations of scenes. Journal of Experimental Psychology: Human Perception and Performance, 30, 988-1015.

Schmidt, B. K., Vogel, E. K., Woodman, G. F., \& Luck, S. J. (2002). Voluntary and automatic attentional control of visual working memory. Perception \& Psychophysics, 64, 754-763.

Schneider, W., Eschman, A., \& Zuccolotto, A. (2002). E-Prime user's guide. Pittsburgh: Psychology Software Tools Inc..

Scholl, B. J. (2000). Attenuated change blindness for exogenously attended items in a flicker paradigm. Visual Cognition, 7, 377-396.

Shiffrin, R. M., \& Steyvers, M. (1997). A model for recognition memory: REM-retrieving effectively from memory. Psychonomic Bulletin \& Review, 4, 145-166.

Silverman, M. E., \& Mack, A. (2006). Change blindness and priming: when it does and does not occur. Consciousness and Cognition, 15, 409-422.

Simons, D. J. (2000). Current approaches to change blindness. Visual Cognition, 7, 1-15.

Simons, D. J., Chabris, C. F., Schnur, T., \& Levin, D. T. (2002). Evidence for preserved representations in change blindness. Consciousness and Cognition, 11, 78-97.

Simons, D. J., Franconeri, S. L., \& Reimer, R. L. (2000). Change blindness in the absence of a visual disruption. Perception, 29, 1143-1154.

Simons, D. J., \& Rensink, R. A. (2005). Change blindness: past, present, and future. Trends in Cognitive Sciences, 9, 16-20.

Smilek, D., Eastwood, J. D., \& Merikle, P. M. (2000). Does unattended information facilitate change detection? Journal of Experimental Psychology: Human Perception and Performance, 26, 480-487.

Smith, D. G., \& Duncan, M. J. J. (2004). Testing theories of recognition memory by predicting performance across paradigms. Journal of Experimental Psychology: Learning, Memory, and Cognition, 30, 615-625.

Snodgrass, J. G., \& Hirshman, E. (1994). Dissociations among implicit and explicit memory tasks: the role of stimulus similarity. Journal of Experimental Psychology: Learning, Memory, and Cognition, 20, $150-160$.

Stark, C. E. L., \& McClelland, J. L. (2000). Repetition priming of words, pseudowords, and nonwords. Journal of Experimental Psychology: Learning, Memory, and Cognition, 26, 945-972.

VanRullen, R., \& Koch, C. (2003). Competition and selection during visual processing of natural scenes and objects. Journal of Vision, 3, $75-85$.

Varakin, D. A., \& Levin, D. (2006). Change blindness and visual memory: visual representations get rich and act poor. British Journal of Psychology, 97, 51-77.

Wilken, P., \& Ma, W. J. (2004). A detection theory account of change detection. Journal of Vision, 4, 1120-1135.

Williams, P., \& Simons, D. J. (2000). Detecting changes in novel, complex three-dimensional objects. Visual Cognition, 7, 297-322.

Yang, C.-T., Hung, Y.-C., \& Yeh, Y.-Y. (2003). Perceived similarity among colored objects. Unpublished raw data.

Yeh, Y.-Y., Yang, C.-T., \& Chiu, Y.-C. (2005). Binding or prioritization: the role of attention in visual short-term memory. Visual Cognition, 12, 759-799.

Zeelenberg, R. (2005). Encoding specificity manipulations do affect retrieval from memory. Acta Psychologica, 119, 107-121.

Zelinsky, G. (2003). Detecting changes between real-world objects using spatio-chromatic filters. Psychonomic Bulletin \& Review, 10, 533-555. 